\title{
Pair Interactions among Ternary DPPC/POPC/Cholesterol Mixtures in Liquid-Ordered and Liquid-Disordered Phases $^{\dagger}$
}

\begin{abstract}
Jing Yang, ${ }^{a}$ Jordi Martí, ${ }^{* a}$ and Carles Calero ${ }^{* b}$
Saturated phospholipids, unsaturated phospholipids, and cholesterol are essential components of cell membranes, making the understanding of their mutual interactions of great significance. We have performed microsecond molecular dynamics simulations on ternary mixtures of DPPC/ $\mathrm{POPC} /$ cholesterol to systematically examine lipid-lipid and cholesterol-lipid interactions in the liquid-ordered and the liquid-disordered phases. The results show that there exists a competition between tighter packing of cholesterol-lipid and looser packing of lipid-lipid as the membrane changes from the liquid-disordered phase to liquid-ordered phase. Depending on the lipid saturation, the favor of lipid-lipid interactions is in the order of saturated-saturated $>$ monounsaturatedmonounsaturated $>$ saturated-monounsaturated. Cholesterol-saturated lipid interactions are more favorable than cholesterol-monounsaturated lipid ones. The results are consistent with the push-pull forces derived from experiments and give general insights on the interactions among membrane components.
\end{abstract}

\section{Introduction}

Cholesterol is an essential component of cell membranes playing a central role in maintaining the structure of the membranes and regulating their functions ${ }^{1,2}$. Mixtures of phospholipids and cholesterol form liquid-disordered $\left(l_{d}\right)$ and liquid-ordered $\left(l_{o}\right)$ phases at different cholesterol concentrations. While the $l_{o}$ state has been considered as a good working model for lipid rafts, the $l_{d}$ state mimics well the fluid, liquid crystalline phase of lipid membranes ${ }^{3,4}$. Study of the interactions of lipid-lipid and cholesterollipid in both $l_{d}$ and $l_{o}$ phases is important for further understanding of cell membranes ${ }^{5,6}$.

Saturated and unsaturated phospholipids interact differently with each other and with cholesterol in different phases. The diversity of their mutual interactions determines their spatial distribution in the membrane ${ }^{5}$. Regen et al. provided the first direct experimental measurements of the interactions between the saturated lipid and cholesterol and also between the monounsaturated lipid and cholesterol in the $l_{d}$ and $l_{o}$ phases using exchangeable mimics of 1,2dipalmitoyl-sn-glycero-3-phosphocholine (DPPC), 1-palmitoyl2-oleoyl-sn-glycero-3-phosphocholine (POPC), and cholesterol. They showed that DPPC and cholesterol exhibit strong attraction

\footnotetext{
${ }^{a}$ Department of Physics and Nuclear Engineering, Technical University of CataloniaBarcelona Tech, B4-B5 Northern Campus, Jordi Girona 1-3, 08034 Barcelona, Catalonia, Spain; E-mail: jordi.marti@upc.edu

${ }^{b}$ Center for Polymer Studies and Department of Physics, Boston University, 590 Commonwealth Avenue, Boston, Massachusetts 02215, United States; E-mail: ccalero@bu.edu

$\dagger$ Electronic Supplementary Information (ESI) available
}

in the $l_{o}$ phase but mix ideally in the $l_{d}$ phase, and that POPC and cholesterol exhibit significant repulsion in the $l_{d}$ phase but mix ideally in the $l_{o}$ phase ${ }^{3,7}$. More recently, they measured the interactions between DPPC and POPC and found that such interactions are significantly repulsive in the $l_{d}$ phase but are neither attractive nor repulsive in the $l_{o}$ phase $^{8}$.

The interactions of lipid-lipid and lipid-cholesterol are nontrivial properties depending on the lipid saturation and also on the mixture phase. Most of the simulations studying such interactions are based on binary mixtures, due to the difficulties of reaching equilibrium in ternary mixtures ${ }^{6}$. However, since cell membranes are composed of saturated lipids, unsaturated lipids, and cholesterol, study on ternary mixtures can give more ideal descriptions of such mutual interactions. In this paper, we perform microsecond all-atom molecular dynamics (MD) simulations on the ternary mixtures of DPPC/POPC/cholesterol in $l_{d}$ and $l_{o}$ phases. The results are consistent with available experiments and give systematical descriptions of the mutual interactions between various like or unlike species in different phases, which could be helpful to deepen our understanding of lipid rafts ${ }^{9}$.

\section{Methods}

Two lipid bilayer systems were generated by means of the CHARMM-GUI web-based tool ${ }^{10,11}$ to simulate membranes in the $l_{d}$ and $l_{o}$ phases. Each system has $128 \mathrm{lipid} /$ cholesterol molecules and 5120 TIP3P water molecules ${ }^{12}$, corresponding to a hydration number of 40 water per lipid. The proportions of various lipid species are carefully chosen to represent DPPC/POPC/cholesterol ternary mixtures in different phases ${ }^{13,14}$. We prepared a bilayer 
in the $l_{o}$ phase using 36 DPPC, 36 POPC, and 56 cholesterol (CHOL) molecules, which corresponds to a cholesterol concentration of 44\%. In addition, we prepared a bilayer consisting of 52 DPPC, 52 POPC, and 24 CHOL, corresponding to a cholesterol concentration of $19 \%$, which exhibits a more disordered $l_{d}$ phase. MD simulations were performed using NAMD $2.9^{15}$ and the CHARMM36 force field ${ }^{16,17}$. Each bilayer system was equilibrated for $100 \mathrm{~ns}$ in the NPT ensemble at $1 \mathrm{~atm}$ and 303 K. A time step of 2 fs was used. Covalent bonds with hydrogen atoms of lipids were kept rigid using SHAKE ${ }^{18}$, and water molecules were kept rigid using SETTLE ${ }^{19}$. The particle mesh Ewald method was employed to compute long-range electrostatic interactions ${ }^{20}$. The cutoff for Lennard-Jones interactions was set to $12 \AA$ and smoothly switched from $10 \AA$. Pressure was controlled by the Langevin piston Nosé-Hoover method ${ }^{21}$. Temperature was controlled by the Langevin dynamics with a damping coefficient of $1 \mathrm{ps}^{-122}$. After equilibration, a 900 ns production run was performed in the NVT ensemble for each bilayer system.

\section{Results and discussion}

The deuterium order parameter $\mathrm{S}_{\mathrm{CD}}$ of the lipid acyl tails is an important property to characterize the order of the lipid bilayer ${ }^{23}$. The order parameter for each $\mathrm{CH}_{2}$ group in the lipid tails is defined as

$$
\mathrm{S}_{\mathrm{CD}}=\frac{1}{2}\left(3<\cos ^{2} \theta>-1\right)
$$

where $\theta$ is the angle between a $\mathrm{C}-\mathrm{H}$ vector and the bilayer normal, and the angular brackets denote both ensemble and time average. The resulting $\left|\mathrm{S}_{\mathrm{CD}}\right|$ obtained from the bilayer systems considered, with $19 \%$ and $44 \%$ cholesterol, are shown in Figure 1 . For both sn-1 and sn-2 chains of DPPC, the order parameters increase significantly (Figure 1a). The same trends are also observed for both chains of POPC with relatively small order parameters in the middle of the sn-2 unsaturated chain due to the double bond (Figure 1b). These results indicate an ordering of the lipid bilayer with increasing cholesterol content, comparable with a liquid-disordered $\left(l_{d}\right)$ to a liquid-ordered $\left(l_{o}\right)$ phase transition in binary systems ${ }^{24-27}$.

The packing of lipid-lipid and cholesterol-lipid pairs in the $l_{d}$ and the $l_{o}$ phases are characterized by the lateral radial pair distribution function $g(r)$, where $r$ is the projected distance in the lateral plane between centers of mass (COM) of two molecules ${ }^{28,29}$. As indicated by the decrease in the first peak of $g(r)$ in Figure $2 \mathrm{a}$, the packing of DPPC-DPPC becomes looser when the bilayer changes from the $l_{d}$ phase to the $l_{o}$ phase. In contrast, the packing of POPC-POPC is almost not affected by the phases (Figure $2 \mathrm{~b}$ ). For DPPC-POPC, the packing also becomes looser in the $l_{o}$ phase since the position of the first peak of $g(r)$ has been shifted far away (Figure 2c). The looser packing for DPPC-DPPC and DPPC-POPC and the unaffected packing for POPC-POPC in the $l_{o}$ phase seems in contradiction with most of the experimental and computational results that lipid bilayers become more condensed and more tightly packed at higher cholesterol concentration ${ }^{2,6}$. However, we should remember that there are also pairs involving cholesterol in the ternary bilayer systems. For CHOL-DPPC, the first peak of $g(r)$ becomes more apparent and increase significantly in the $l_{o}$ phase, indicating a more condensed packing (Figure 2d). For CHOL-POPC, the packing is slightly tighter in the $l_{o}$ phase as the first peak of $g(r)$ becomes more evident (Figure 2e). For CHOL-CHOL, the packing is tighter as the first, second, and third peak of $g(r)$ increase in the $l_{o}$ phase (Figure 2f). Therefore, all the pairs involving CHOL become more tightly packed at higher cholesterol concentration, which compensates the looser packing effects of DPPC-DPPC and DPPC-POPC in the $l_{o}$ phase and indicates a competition between tighter packing of cholesterol-lipid and looser packing of lipid-lipid as the mixtures condense.

In order to compare different kinds of interactions systematically, we calculate the potential of mean force (PMF) for lipidlipid and cholesterol-lipid pairs by

$$
\operatorname{PMF}(r)=-k_{B} T \ln g(r),
$$

where $g(r)$ is the lateral radial pair distribution function ${ }^{30}$. The resulting PMF for lipid-lipid pairs in the $l_{d}$ phase and the $l_{o}$ phase are shown in Figure 3a. In the pair distance of $6 \AA<r<10 \AA$, the lowest profile is DPPC-DPPC in the $l_{d}$ phase, indicating that interactions among saturated phospholipids in the $l_{d}$ phase are most favorable. The highest profile is DPPC-POPC in the $l_{d}$ phase, suggesting that interactions between saturated phospholipids and monounsaturated phospholipids in the $l_{d}$ phase are most unfavorable, which is consistent with the push force measured by Regen et al. ${ }^{8}$ The resulting PMF for the pairs involving cholesterol are shown in Figure $3 \mathrm{~b}$. In $6 \AA<r<10 \AA$, there is a clear minimum for CHOL-DPPC in the $l_{o}$ phase compared to the other cholesterollipid interactions, which corresponds to the strong pull proposed by Regen et al. ${ }^{8}$ The interactions for CHOL-POPC in either the $l_{d}$ phase or the $l_{o}$ phase are clearly unfavorable with respect to the strong pull of CHOL-DPPC in the $l_{o}$ phase, which can be attributed to the significant push between cholesterol and monounsaturated phospholipids $^{3,8}$. In contract to the other interactions, the PMF for CHOL-CHOL has several local minima keeping the same positions in the $l_{d}$ phase and the $l_{o}$ phase, which indicates that the favorable packing distances among cholesterol are not affected by the phases.

The interactions between DPPC, POPC and CHOL are not very intense. As a consequence, the distance to first neighbors is not well defined (see Figure 2). For this reason, here we have adopted a geometrical definition of first neighbors based on a Voronoi tesselation of the plane of the membrane, as introduced by Pandit et al. ${ }^{31}$ In our case, the centers of the Voronoi cells are given by the projection on the $\mathrm{XY}$ plane of the center of mass of each of the DPPC, POPC and CHOL molecules. The cell associated to a given center is defined as the region which is closer to that center than to any other. Two molecules are considered to be first neighbors if their corresponding Voronoi cells share an edge. We calculate the number of first neighbors for each DPPC, POPC and CHOL based on Voronoi cells in the $l_{d}$ and $l_{o}$ phases. The probability distribution for the number of first neighbors in Figure 4 shows that DPPC and POPC are more likely to have six first neighbors regardless of the membrane phases. In contrast, the packing 

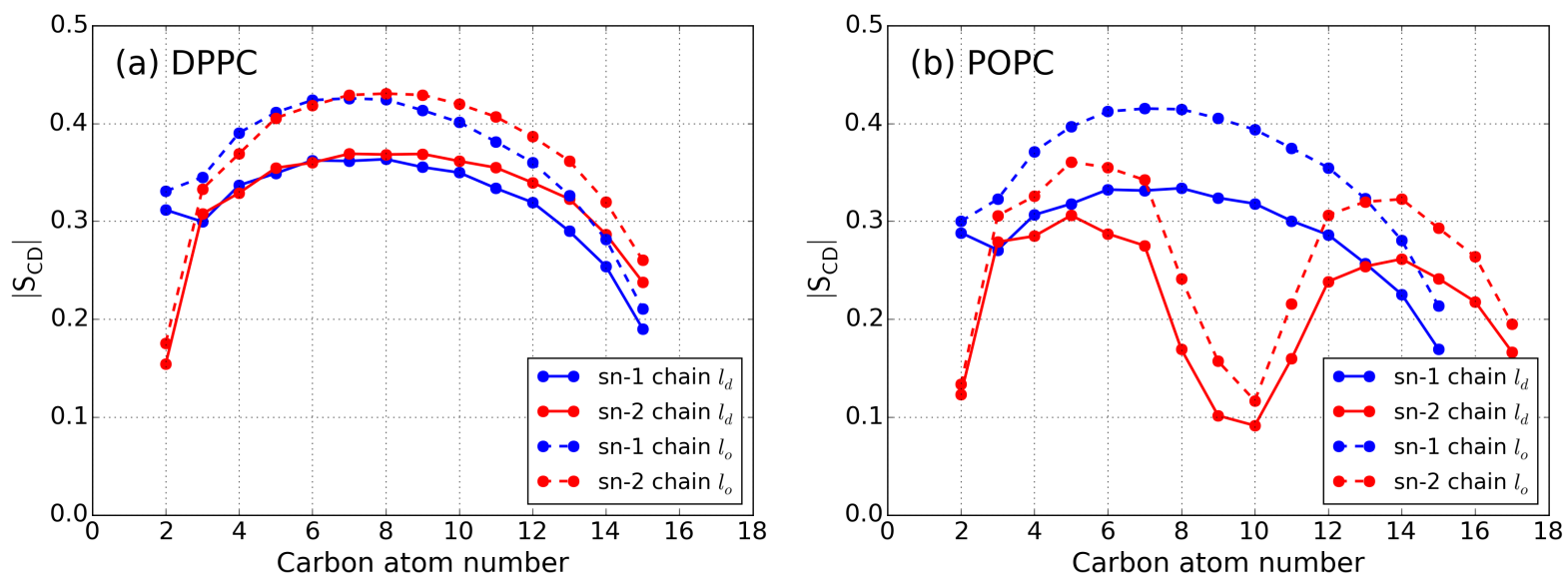

Fig. 1 Order parameter $\left|\mathrm{S}_{\mathrm{CD}}\right|$ for the acyl tails of DPPC (a) and POPC (b) in the liquid-disordered phase (with 19\% cholesterol) and the liquid-ordered phase (with $44 \%$ cholesterol).
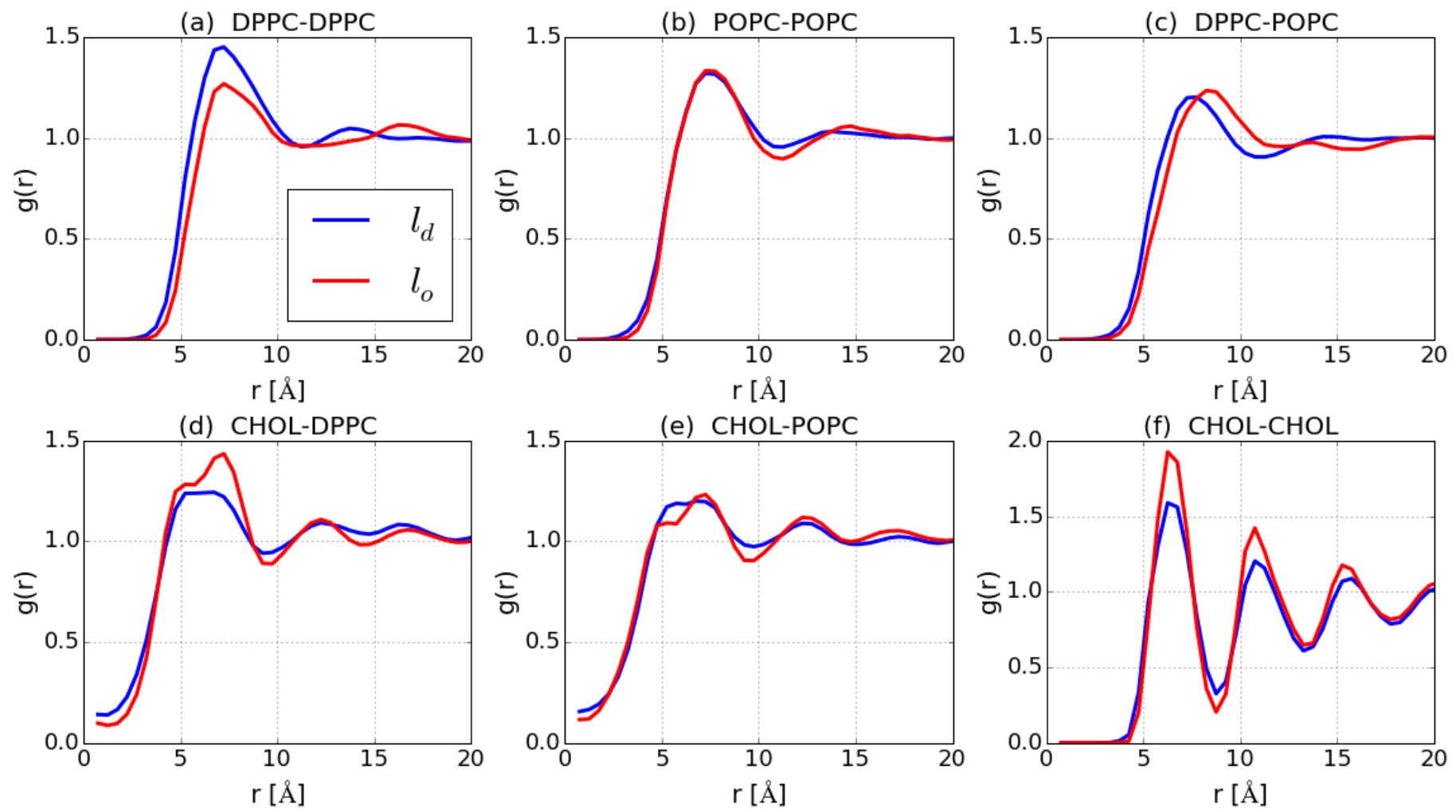

Fig. 2 Lateral radial pair distribution function $g(r)$ for DPPC/POPC/CHOL mixtures in the $l_{d}$ and $l_{o}$ phases.

of first neighbors around CHOL molecules changes with membrane phases. A CHOL molecule will have most likely six first neighbors in the $l_{o}$ phase but five first neighbors in the $l_{d}$ phase. Given the usual hexagonal local order of lipid or cholesterol units in cell membranes, this fact is quite remarkable. Considering the large proportion of CHOL molecules in membranes of the $l_{o}$ phase ( $44 \%$ in our case), the changes of packing around CHOL can have an impact on the interactions among membrane components.

Another way to look at the lipid-lipid and lipid-cholesterol pairing is by obtaining the corresponding effective association constants. Two kinds of different lipids A and B can form three kinds of first neighbor pairs, i.e. $\mathrm{AA}, \mathrm{BB}$, and $\mathrm{AB}$. The mutual interactions between lipids $\mathrm{A}$ and $\mathrm{B}$ in equilibrium can be characterised by an effective association constant, $\mathrm{K}$, defined by

$$
K=\frac{[A B]^{2}}{[A A][B B]},
$$

where $[\mathrm{AA}],[\mathrm{BB}]$, and $[\mathrm{AB}]$ stand for the number of first neighbor pairs of each class. Random mixing is achieved when $\mathrm{K}=$ 4.0 , repulsions are indicated by $\mathrm{K}<4.0$, and attractions are indicated by $\mathrm{K}>4.0$. To quantitatively reveal the pair interactions among various membrane components, we calculate the effective 

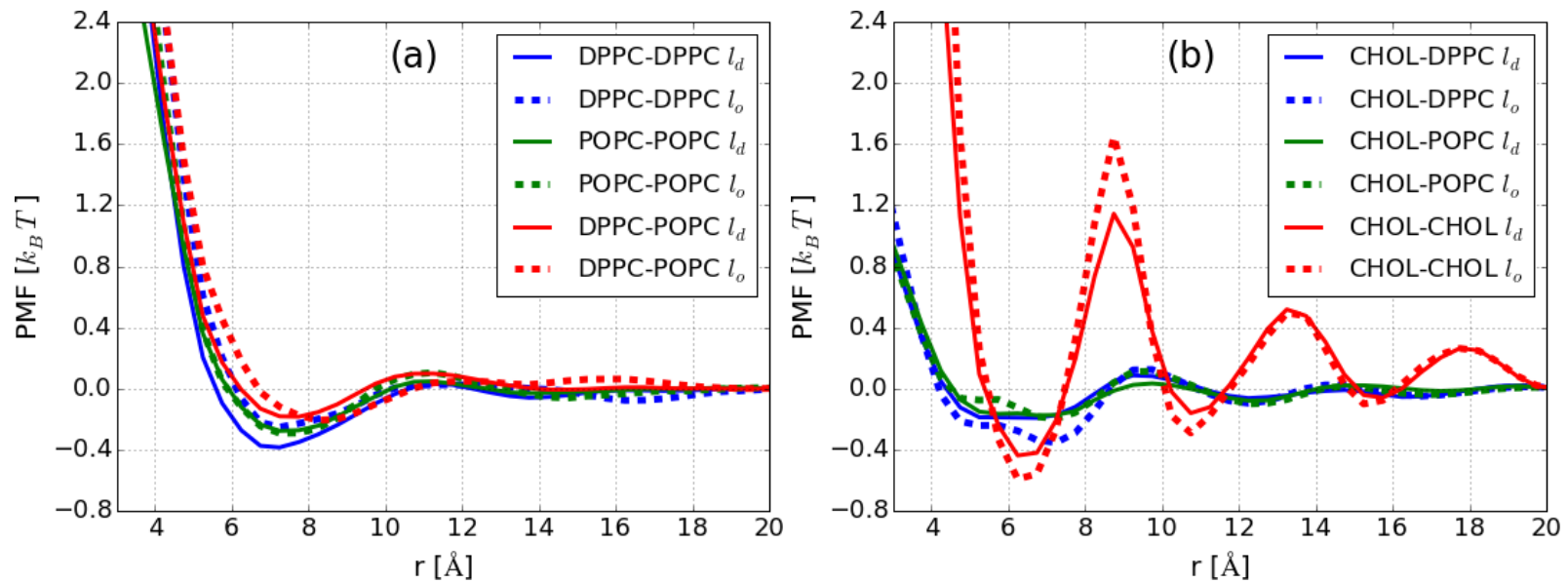

Fig. 3 Potential of mean force (PMF) for lipid-lipid (a) and cholesterol-lipid (b) pairs in the $l_{d}$ (solid line) and the $l_{o}$ (dash line) phases.

association constant $\mathrm{K}$ among different species in our simulation and compare it with analogous equilibrium constants obtained in experiment under similar conditions ${ }^{8}$. The association constant $\mathrm{K}$ for DPPC-POPC interactions shows an increase from less than 4.0 in the $l_{d}$ phase to more than 4.0 in the $l_{o}$ phase, which qualitatively agrees with experiments and indicates the repulsion in the $l_{d}$ phase and the attraction in the $l_{o}$ phase. The increase of $\mathrm{K}$ is also observed in the case of CHOL-DPPC interactions, corresponding to a strong attraction in the $l_{o}$ phase. Finally, for the case of CHOL-POPC interactions, we also obtain a good qualitative agreement with experiments in the $l_{o}$ phase, whereas in the $l_{d}$ phase the simulation result of $\mathrm{K}=6.2$ is not in agreement with experiment, which reveals a repulsive interaction ${ }^{8}$. The difference with experiment could be due to the difference in composition of the membrane used in the simulations, which could alter the subtle balance between push and pull forces which define lipid pairings.

Table 1 Effective association constants $\mathrm{K}$ for various pair interactions in the $l_{d}$ and $l_{o}$ phases obtained from our simulations.

\begin{tabular}{|c|c|c|}
\hline interactions & phase & $\mathrm{K}$ \\
\hline DPPC-POPC & $l_{d}$ & $3.8 \pm 0.1$ \\
& $l_{o}$ & $5.2 \pm 0.4$ \\
\hline CHOL-DPPC & $l_{d}$ & $5.3 \pm 0.4$ \\
& $l_{o}$ & $6.6 \pm 0.2$ \\
\hline CHOL-POPC & $l_{d}$ & $6.2 \pm 0.3$ \\
& $l_{o}$ & $4.3 \pm 0.2$ \\
\hline
\end{tabular}

\section{Conclusions}

We have systematically examined the mutual interactions among saturated phospholipids, monounsaturated phospholipids, and cholesterol in the liquid-ordered and the liquid-disordered phases by performing microsecond $\mathrm{MD}$ simulations on the ternary mixtures of DPPC/POPC/cholesterol. The results show that there exists a competition between tighter packing of cholesterol-lipid and looser packing of lipid-lipid as the membrane changes from the liquid-disordered phase to liquidordered phase. Depending on the lipid saturation, the fa- vor of lipid-lipid interactions is in the order of saturatedsaturated $>$ monounsaturated-monounsaturated $>$ saturatedmonounsaturated. The cholesterol-saturated lipid interaction is more favorable than cholesterol-monounsaturated lipid interaction, though the push force of the latter one reduces significantly as the mixtures condense. The results are in overall good agreement with the pull-push forces derived from experiments and give general insights on the interactions among membrane components.

\section{Acknowledgement}

The authors acknowledge computer resources and assistance provided by the Red Española de Supercomputación, through the Centre de Supercomputació de Catalunya. J.Y. acknowledges support from a FI-AGAUR fellowship of the Generalitat de Catalunya. J.M. gratefully acknowledges financial support from the Direcció General de Recerca de la Generalitat de Catalunya (Grant 2009-SGR1003) and the Spanish MINECO for grant FIS2012-39443-C0201. C.C. acknowledges support from the Beatriu de Pinós program (BP-DGR 2011).

\section{References}

1 H. Ohvo-Rekilä, B. Ramstedt, P. Leppimäki and J. Peter Slotte, Progress in Lipid Research, 2002, 41, 66-97.

2 T. P. W. McMullen, R. N. a. H. Lewis and R. N. McElhaney, Current Opinion in Colloid and Interface Science, 2004, 8, 459468.

3 M. R. Krause, T. a. Daly, P. F. Almeida and S. L. Regen, Langmuir, 2014, 30, 3285-3289.

4 H.-J. Kaiser, D. Lingwood, I. Levental, J. L. Sampaio, L. Kalvodova, L. Rajendran and K. Simons, Proceedings of the National Academy of Sciences of the United States of America, 2009, 106, 16645-16650.

5 P. F. F. Almeida, Biochimica et Biophysica Acta - Biomembranes, 2009, 1788, 72-85.

6 M. L. Berkowitz, Biochim. Biophys. Acta, 2009, 1788, 86-96.

7 S. Turkyilmaz, P. F. Almeida and S. L. Regen, Langmuir, 2011, 27, 14380-14385. 
(a) Disordered

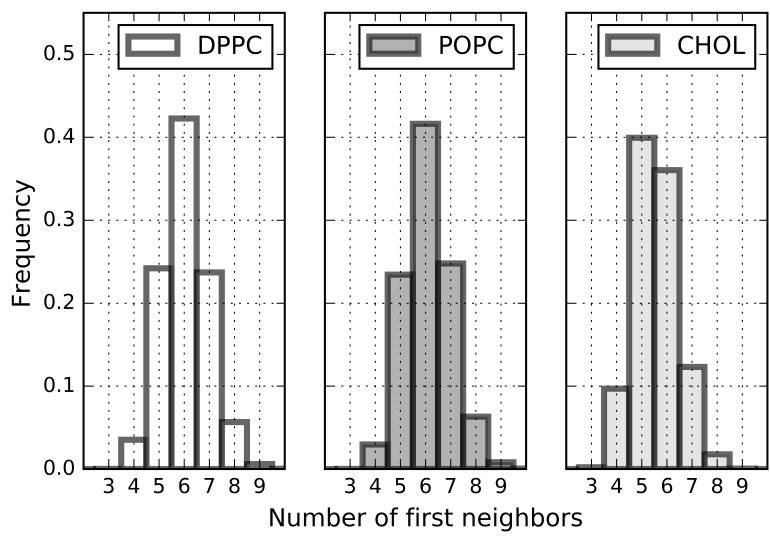

(b) Ordered

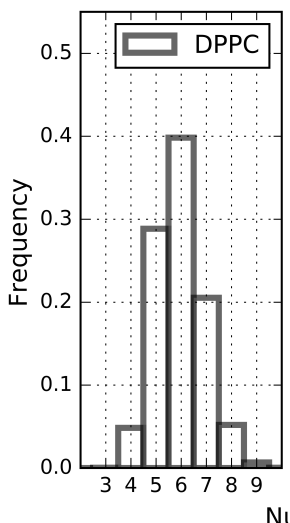

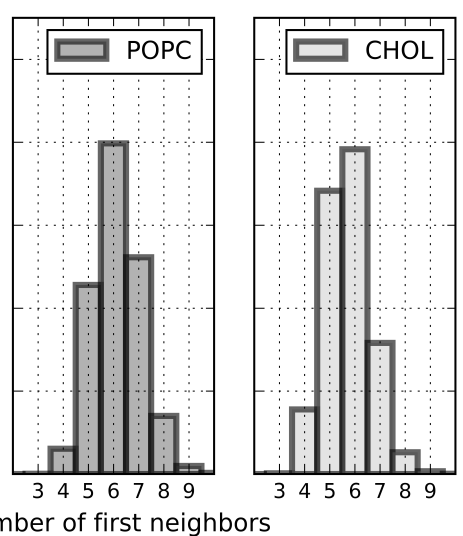

Fig. 4 The probability distribution for the number of first neighbors around DPPC, POPC and CHOL in the $l_{d}$ phase (a) and the $l_{o}$ phase (b).

8 C. Wang, M. R. Krause and S. L. Regen, Journal of the American Chemical Society, 2015, 137, 664-666.

9 L. J. Pike, Journal of Lipid Research, 2009, 50, S323-S328.

10 S. Jo, T. Kim, V. G. Iyer and W. Im, J. Comput. Chem., 2008, 29, 1859-1865.

11 S. Jo, J. B. Lim, J. B. Klauda and W. Im, Biophys. J., 2009, 97, 50-58.

12 W. L. Jorgensen, J. Chandrasekhar, J. D. Madura, R. W. Impey and M. L. Klein, J. Chem. Phys., 1983, 79, 926-935.

13 J. Zhao, J. Wu, H. Shao, F. Kong, N. Jain, G. Hunt and G. Feigenson, Biochimica et Biophysica Acta (BBA) - Biomembranes, 2007, 1768, 2777 - 2786.

14 D. Marsh, Biochimica et Biophysica Acta (BBA) - Biomembranes, 2009, 1788, $2114-2123$.

15 J. C. Phillips, R. Braun, W. Wang, J. Gumbart, E. Tajkhorshid, E. Villa, C. Chipot, R. D. Skeel, L. Kalé and K. Schulten, J. Comput. Chem., 2005, 26, 1781-1802.

16 J. B. Klauda, R. M. Venable, J. A. Freites, J. W. O'Connor, D. J. Tobias, C. Mondragon-Ramirez, I. Vorobyov, A. D. MacKerell and R. W. Pastor, J. Phys. Chem. B, 2010, 114, 7830-7843.

17 J. B. Lim, B. Rogaski and J. B. Klauda, J. Phys. Chem. B, 2012, 116, 203-210.

18 J.-P. Ryckaert, G. Ciccotti and H. J. Berendsen, J. Comput. Phys., 1977, 23, 327-341.

19 S. Miyamoto and P. A. Kollman, J. Comput. Chem., 1992, 13,
952-962.

20 U. Essmann, L. Perera, M. L. Berkowitz, T. Darden, H. Lee and L. G. Pedersen, J. Chem. Phys., 1995, 103, 8577.

21 W. Hoover, Phys. Rev. A, 1985, 31, 1695-1697.

22 S. E. Feller, Y. Zhang, R. W. Pastor and B. R. Brooks, J. Chem. Phys., 1995, 103, 4613.

23 L. S. Vermeer, B. L. De Groot, V. Réat, A. Milon and J. Czaplicki, European Biophysics Journal, 2007, 36, 919-931.

24 C. Hofsäss, E. Lindahl and O. Edholm, Biophysical journal, 2003, 84, 2192-2206.

25 a. M. Smondyrev and M. L. Berkowitz, Biophysical journal, 1999, 77, 2075-2089.

26 T. Róg and M. Pasenkiewicz-Gierula, Biochimie, 2006, 88, 449-460.

27 S. a. Pandit, S. W. Chiu, E. Jakobsson, A. Grama and H. L. Scott, Langmuir, 2008, 24, 6858-6865.

28 C. Hong, D. P. Tieleman and Y. Wang, Langmuir : the ACS journal of surfaces and colloids, 2014, 30, 11993-2001.

29 K. Huang and A. E. García, Journal of chemical theory and computation, 2014, 10, 4264-4272.

30 J. Timko, A. De Castro and S. Kuyucak, The Journal of Chemical Physics, 2011, 134, 204510.

31 S. A. Pandit, D. Bostick and M. L. Berkowitz, J. Chem. Phys., 2003, 119, 2199-2205. 